\title{
Successful management of prostate cancer with bulky pelvic lymph node metastases after rapid development of castration-resistant prostate cancer: a case report with review of the literature
}

\author{
Lixin Mai ${ }^{1}$, Yonghong $\mathrm{Li}^{2}$, Ping Yang ${ }^{3}$, Zitong Zhang ${ }^{1}$, Jianhua $\mathrm{Wu}^{1}$, Fangjian Zhou ${ }^{2}$, Yang Liu ${ }^{1}$, Liru $\mathrm{He}^{1}$ \\ ${ }^{1}$ Department of Radiation Oncology, ${ }^{2}$ Department of Urology Oncology, ${ }^{3}$ Department of Pathology, Sun Yat-Sen University Cancer Center, State \\ Key Laboratory of Oncology in South China, Collaborative Innovation Center for Cancer Medicine, Guangzhou 510060, China \\ Correspondence to: Liru He; Yang Liu. Department of Radiation Oncology, Sun Yat-Sen University Cancer Center, State Key Laboratory of Oncology \\ in South China, Collaborative Innovation Center for Cancer Medicine, No. 651, Dongfeng Road East, Guangzhou 510060, China. \\ Email: helir@sysucc.org.cn; liuyang1@sysucc.org.cn.
}

\begin{abstract}
The rapid development of castration-resistant prostate cancer (CRPC) is a poor prognostic sign for loco-regionally advanced prostate cancer. Non-metastatic CRPC (nmCRPC) with bulky regional lymph node metastases is extremely rare and difficult to treat clinically without reported case. We present a case of a 72-year-old man with locally advanced prostate cancer with bulky lymph node metastases $(53 \mathrm{~mm}$ $\times 77 \mathrm{~mm} \times 67 \mathrm{~mm}$ ), developing nmCRPC after 9-month ADT treatment. Immunohistochemistry (IHC) tests found partially positive MUC1 and negative BRCA1 expression in the initial biopsy specimens. Nextgeneration sequence analysis on the blood specimen after CRPC predicted a good tolerance to docetaxel. According to the multidisciplinary team recommendations, he was administrated docetaxel $75 \mathrm{mg} / \mathrm{m}^{2}$ on day 1 every 21 days for 6 cycles, and subsequently radiotherapy, with the delivery of a total dose of 67.5, 60-65 and 47.5 Gy in 25 fractions to the prostate, the enlarged lymph nodes and the whole pelvis respectively. Over a follow-up of 50 months, his disease has achieved good local control and he is alive without evidence of distant metastases or late adverse events. This case highlights individualized and multimodal therapy of intensification of systemic therapy and timely application of radiotherapy in such rare condition.
\end{abstract}

Keywords: Case report; castration-resistant; chemotherapy; prostate cancer; radiotherapy

Submitted Mar 09, 2020. Accepted for publication May 14, 2020.

doi: $10.21037 /$ tau-20-725

View this article at: http://dx.doi.org/10.21037/tau-20-725

\section{Introduction}

Prostate cancer is the second most common cancer and the fifth leading cause of cancer death seen in males worldwide (1). In China, a steady and rapid increase in both the incidence and the mortality rate of prostate cancer has been observed since 2000. According to the cancer statistics published by National Cancer Center in 2019, the estimated new cases of prostate cancer is 72,000 , while the estimated deaths is 31,000 in China (2). It is reported that patients with regional nodal involvement, who usually have a poorer prognosis for subsequent systemic disease progression (3), can frequently be observed in our daily practice. Androgen deprivation therapy (ADT) and radiotherapy with/without abiraterone is the standard of care (SOC) for regional prostate cancer, but such treatment strategy may not have the similar effect in the setting of bulky lymph node metastases and short PSA doubling time.

It is key to shrink the tumor as much as possible so as to create the opportunity of definitive local treatment for such patients. ADT with or without antiandrogen may be not enough to carry this point. Despite the survival benefit and distant metastasis rate reduction have been reported in 

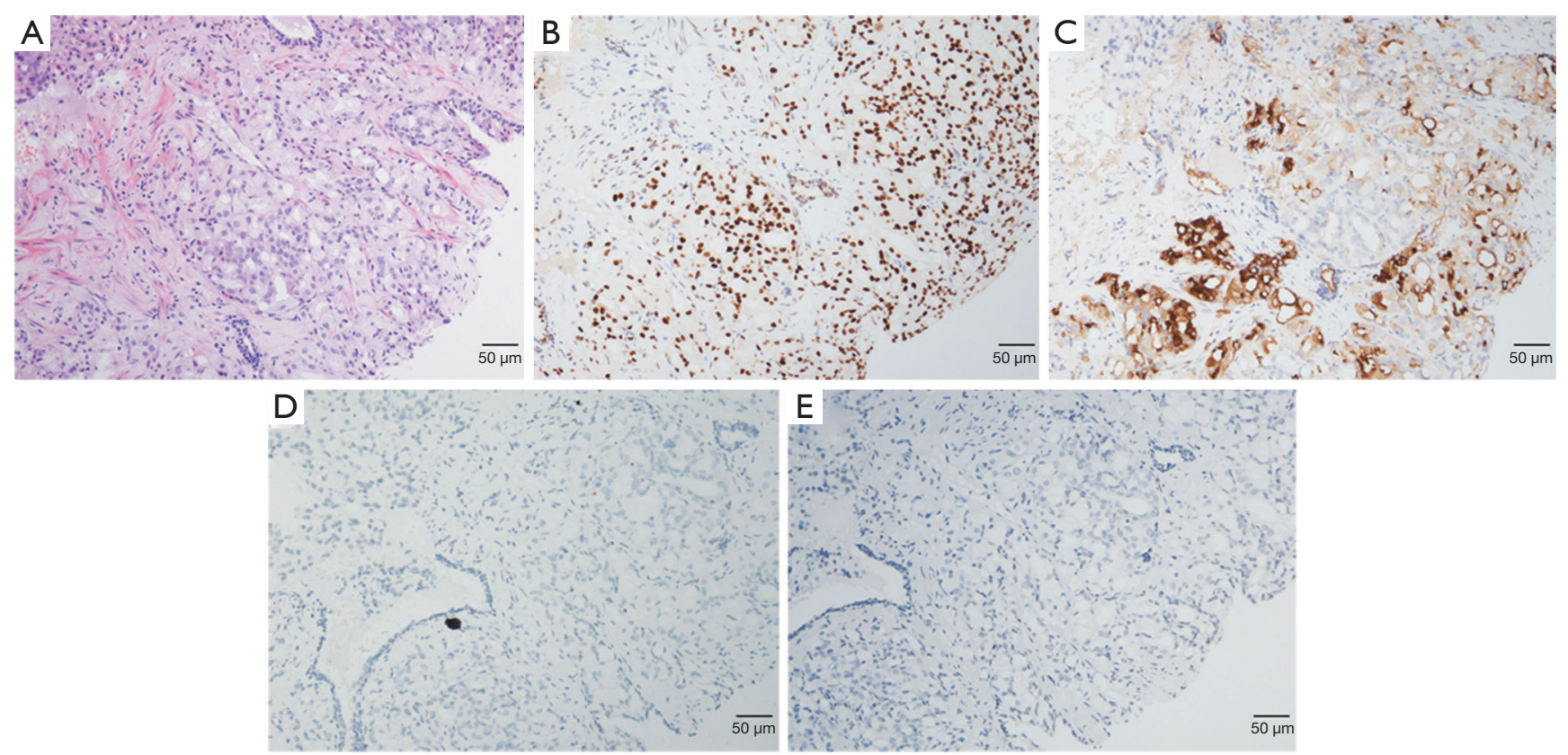

Figure 1 Representative photomicrographs of hematoxylin-eosin and immunohistochemistry staining of the case. (A) Prostate needle biopsy. Microscopic appearance of the prostatic adenocarcinoma with Gleason score: 4+4=8 (ISUP Grade 4); (B) diffuse and strong nuclear immunoexpression of Androgen Receptor (AR) (3+, 95\% positivity); (C) moderated to strong cytoplasmic immunoexpression of MUC1 $(2+\sim 3+, 30 \%$ positivity); lack of expression of AR-V7 (D) and BRCA1 (E). All images are shown as $\times 200$ magnification.

high risk patients, chemotherapy is not yet recommended in those patients partly due to its adverse events (4). There is an estimated average time of 2-3 years from the initiation of ADT to castration-resistant prostate cancer (CRPC) development (5). However, patients with highly malignant tumor who develop rapidly to non-metastatic CRPC (nmCRPC) in only several months are also occasionally observed. Some scholars believe that early use of docetaxel in nmCRPC patients may prolong overall survival (OS) (6). On the other hand, whether there is a role of radiotherapy in nmCRPC is a question that has been long raised. To our knowledge, no result of clinical trials on radiotherapy with/without chemotherapy has been reported in regional nmCRPC patients.

Herein, the following case report presents a patient with locally advanced prostate cancer and bulky lymph node metastases, who rapidly developed nmCRPC after 9-month ADT treatment. His disease has been effectively controlled and the quality of life has been well maintained after chemotherapy plus definitive radiotherapy (7). We present the following case in accordance with the CARE reporting checklist (available at http://dx.doi.org/10.21037/tau-20725).

\section{Case presentation}

A 72-year-old Chinese male presenting with intermittent dysuria for 3 years was admitted into our hospital on December 21st, 2015. His past medical history included hypertension, a 50-year history of smoking and alcohol drinking (250 $\mathrm{g}$ daily). His family history was unremarkable. Physical examination disclosed a mass measuring about $7 \mathrm{~cm}$ in the right lower quadrant of abdomen. Digital rectal examination revealed an enlarged prostate gland of stony-hard consistency. Serum prostate-specific antigen (PSA) was $401.3 \mathrm{ng} / \mathrm{mL}$; testosterone was $27.80 \mathrm{nmol} / \mathrm{L}$. Core biopsy of the prostate proved to be a Gleason 4+4 (ISUP GRADE 4) prostate adenocarcinoma (Figure 1A), with immunohistochemistry (IHC) staining showing AR (androgen receptor)-strong staining intensity (3+), 95\% positive (Figure 1B); MUC1 (mucin 1)-moderate to strong staining intensity $(2+\sim 3+), 30 \%$ positive (Figure 1C); AR-V7 (androgen receptor splice variant 7)-negative (Figure 1D) and BRCA1 (breast cancer 1)-negative (Figure 1E). Bone scan showed no bone metastasis (Figure 2). Pelvic magnetic resonance imaging (MRI) showed enlarged prostate with $44 \mathrm{~mm} \times 30 \mathrm{~mm} \times 35 \mathrm{~mm}$ in size; central lobe and right peripheral zone invasion; seminal vesicle invasion; bilateral 


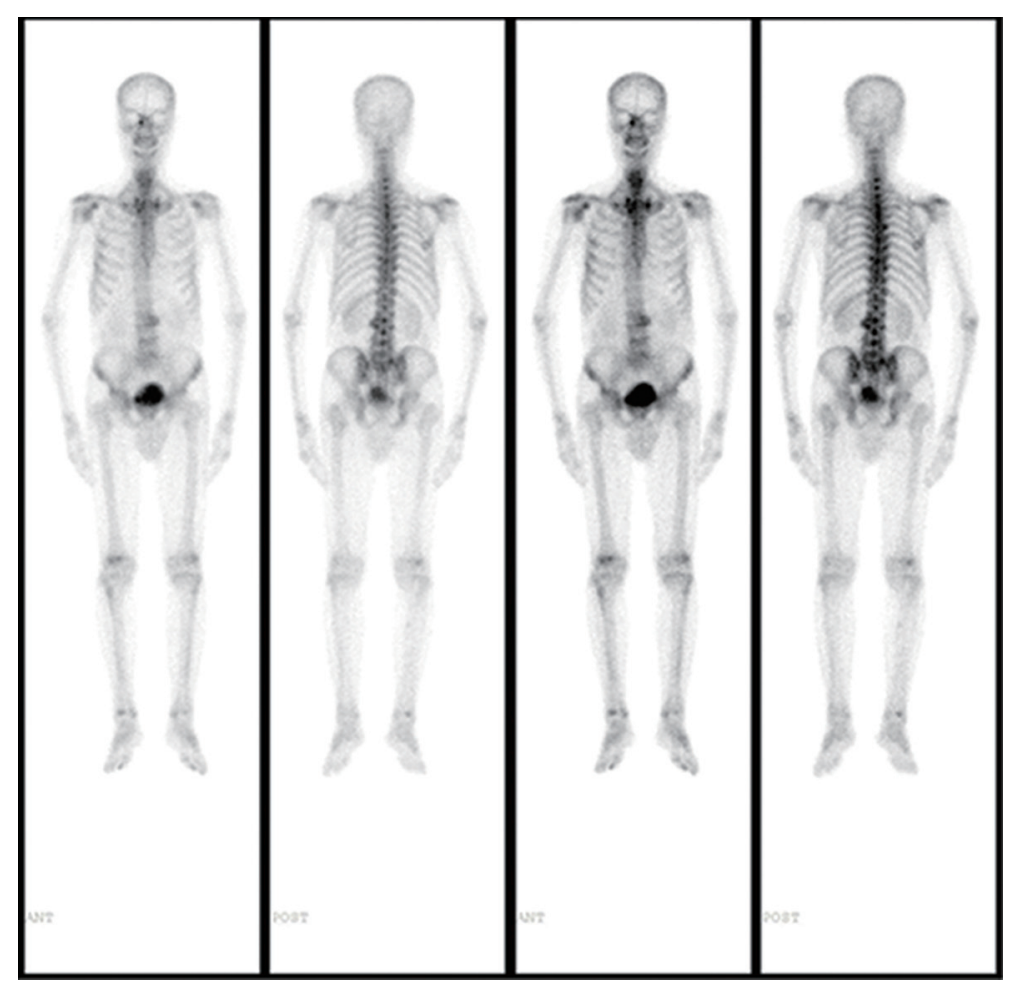

Figure 2 The bone scan images showed no obvious bone metastasis at the time of initial diagnosis.

pelvic lymph node metastases, measuring $53 \mathrm{~mm} \times 77$ $\mathrm{mm} \times 67 \mathrm{~mm}$ and $19 \mathrm{~mm} \times 29 \mathrm{~mm} \times 30 \mathrm{~mm}$ respectively (Figure $3 A$ ). The patient was diagnosed with prostate adenocarcinoma (cT3bN1M0 stage IV) after we ruled out possibilities of other primary tumors supported by the results of laboratory investigations and imaging.

The patient was initially treated with bicalutamide $50 \mathrm{mg}$ daily and goserelin $10.8 \mathrm{mg}$ every 84 days, and his serum testosterone soon reached castration level. A PSA nadir of $13.07 \mathrm{ng} / \mathrm{mL}$ was achieved after 9 months, but soon his PSA continued to rise and no distal metastasis was found (Figure 3B) and nmCRPC developed. Given his rapid progression to nmCRPC, targeted next-generation sequence analysis was performed using post-treatment blood sample to elucidate the potential related genetic variations. However, no gene mutation of clinical significance was found. Chemotherapyrelated polymorphism analysis suggested low toxic effects on docetaxel [CYP4B1 (rs4646487): CC, ERCC1 (rs11615): AG, VAC14 (rs875858): CC].

After the multidisciplinary discussion, the patient received docetaxel $75 \mathrm{mg} / \mathrm{m}^{2}$ every 21 days for 6 cycles, after which his serum PSA decreased to $9.01 \mathrm{ng} / \mathrm{mL}$, and metastatic lymph nodes continually shrank (Figure 3C).
Subsequently, he received image-guided volumetric modulated arc radiotherapy (IGRT-VMAT), with a total dose of $67.5,60-65$ and 47.5 Gy in 25 fractions to the prostate, the enlarged lymph nodes and the whole pelvis, respectively. The treatment was well-tolerated, with only grade 2 neutropenia during chemotherapy and grade 1 acute rectal hemorrhage after radiotherapy.

His serum PSA decreased to 4.64, 1.78, 0.869 and $0.428 \mathrm{ng} / \mathrm{mL}, 1,4,10$ and 22 months after radiotherapy respectively, and the sizes of primary tumor and metastatic lymph nodes continued to decrease (Figure 3D). At the time of this report, 34 months after radiotherapy and 50 months after diagnosis, the patient remains well and is able to do farm work. The pelvic MRI shows no sign of tumor in the prostate and the previous bulky lymph node has shrunk to only $8 \mathrm{~mm}$ (Figure $3 E$ ). Bone scan indicates no sign of metastasis (Figure 4). His PSA keeps declining and has reached $0.008 \mathrm{ng} / \mathrm{mL}$ (Figure 5). The timeline picture was shown in Figure 6.

\section{Discussion}

Prostate cancer is primarily an androgen-driven disease, 

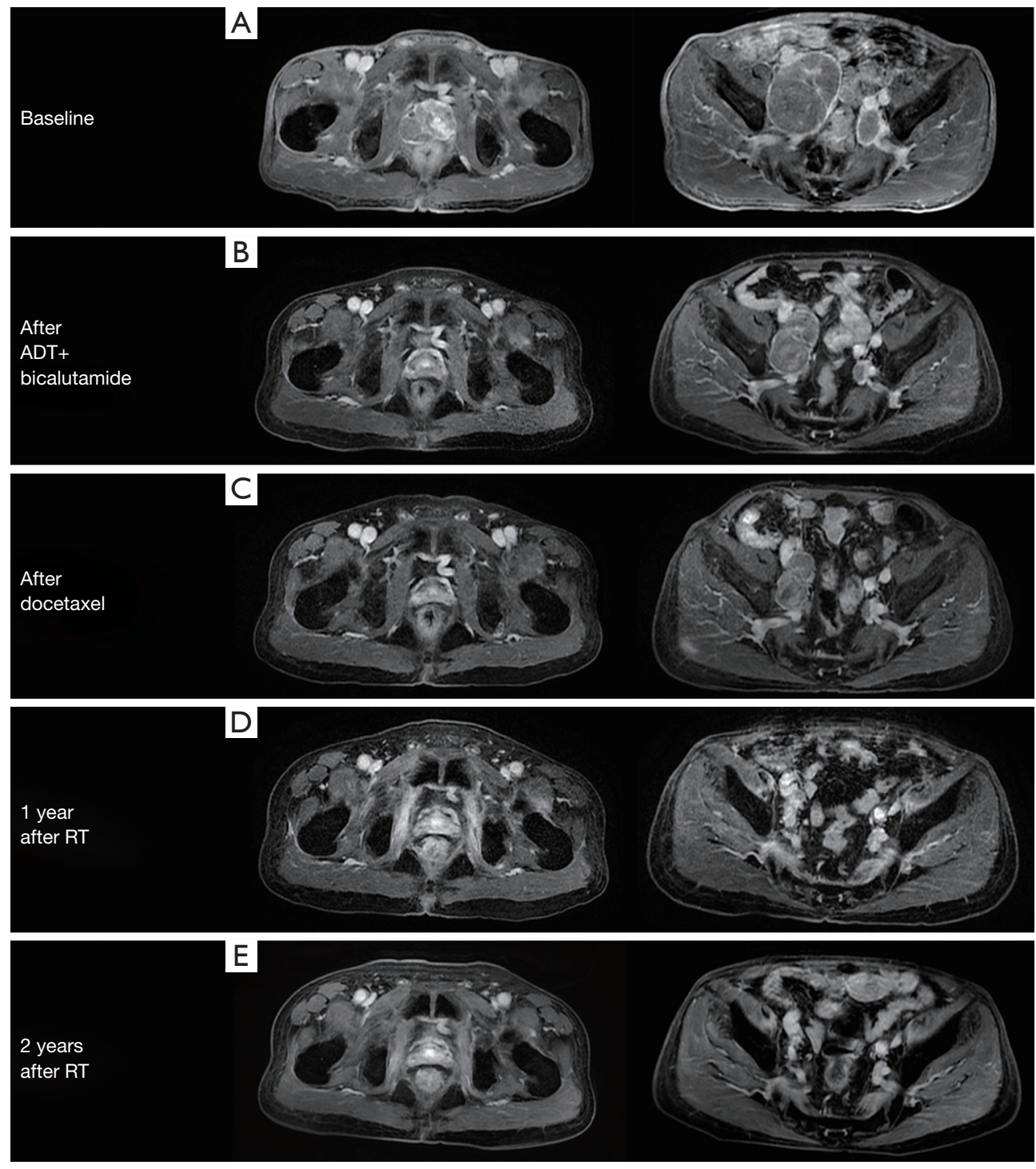

Figure 3 Shrinkage of primary tumor and metastatic lymph nodes after the sequential therapy. Contrast enhanced axial T1-weighted MR image of the pelvis showed (A) the baseline situation of the prostate mass $(44 \mathrm{~mm} \times 30 \mathrm{~mm})$ and bulky metastatic lymph nodes $(53 \mathrm{~mm} \times 77 \mathrm{~mm}$, $19 \mathrm{~mm} \times 29 \mathrm{~mm})$; (B) obvious prostate mass regression $(17 \mathrm{~mm} \times 14 \mathrm{~mm})$ and slight metastatic lymph node reduction $(56 \mathrm{~mm} \times 34 \mathrm{~mm}$, $16 \mathrm{~mm} \times 13 \mathrm{~mm})$ after 9-month ADT treatment; (C) continual metastatic lymph nodes reduction $(51 \mathrm{~mm} \times 27 \mathrm{~mm}, 15 \mathrm{~mm} \times 9 \mathrm{~mm})$ after 6 cycles of docetaxel chemotherapy; (D) further metastatic lymph nodes reduction $(25 \mathrm{~mm} \times 16 \mathrm{~mm}, 12 \mathrm{~mm} \times 7 \mathrm{~mm}) 1$ year after prostate and lymph nodes radiotherapy with previous prostate nodule disappearing; (E) invisible prostate tumor and dramatically shrunk lymph nodes (larger one: $8 \mathrm{~mm} \times 6 \mathrm{~mm}$ ) 2 years after radiotherapy.

and castration-resistance is often driven by AR signaling reactivation. According to the current guidelines, secondary hormone therapy is SOC for nmCRPC, neither chemotherapy nor radiotherapy has been recommended to treat nmCRPC patients. We report a rare case of locally advanced prostate cancer with bulky regional lymph node metastases and high initial PSA level, which obtained dramatic response to chemotherapy and subsequent radiotherapy after rapid progression on $\mathrm{ADT}$ and antiandrogen.

The prognosis of CRPC patients with lymph node metastases is poor, the median OS of which is estimated 


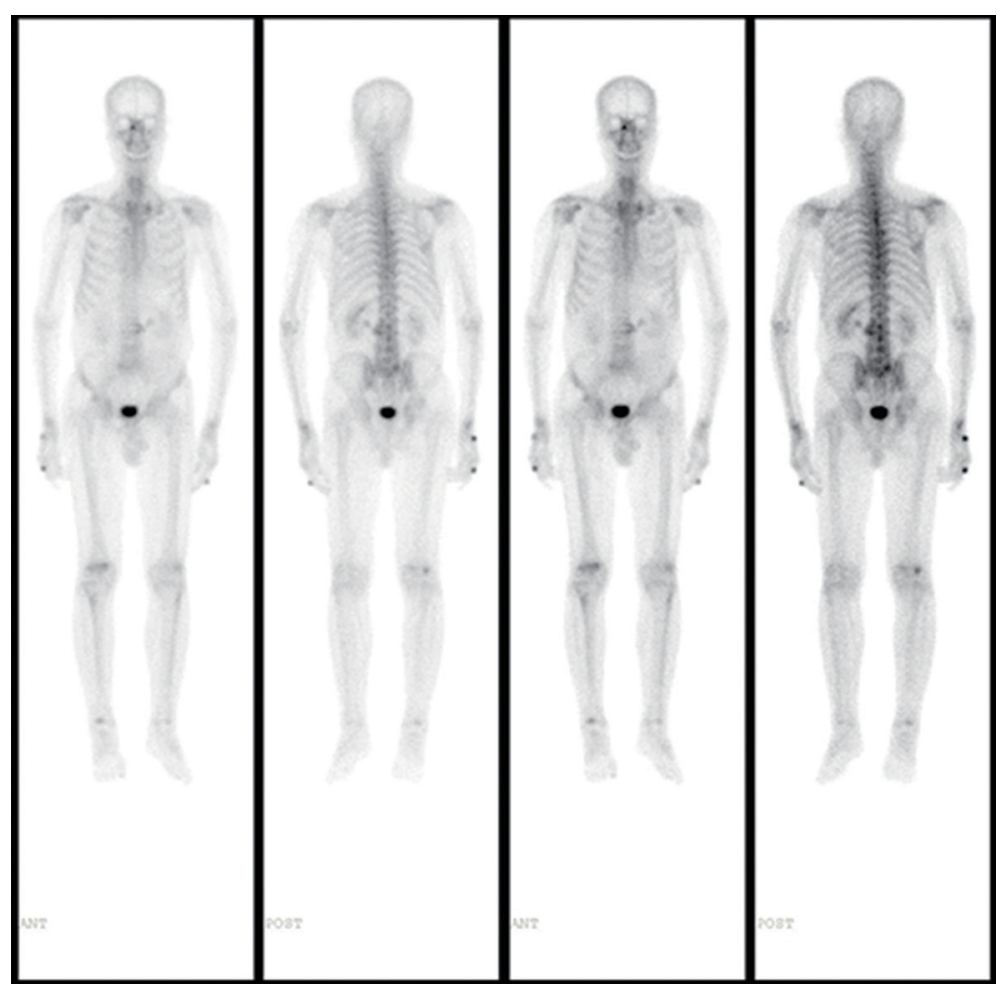

Figure 4 The latest bone scan images showed no sign of bone metastasis at the time of this report.

only 31.6 months (8). Apalutamide and enzalutamide are preferred to treat nmCRPC for the encouraging results from the PROSPER trial and the SPARTAN trial, and earlier use of androgen receptor-axis-targeted drugs (ARAT) may improve OS, PFS, and PSA response (9). However, the PROSPER trial does not clarify the nodal status of the patients enrolled, while the SPARTAN trial excludes patients with lymph nodes more than $2 \mathrm{~cm}$. Besides, it is believed that patients with prior short duration of response to ADT may present with unfavorable response to next generation ARAT in CRPC. Among patients with less than 12 months to CRPC, only $16 \%$ patients respond to ARAT, and the median PFS is only 2.8 months even when enzalutamide is given (10). In this particular case, our patient presented with bulky lymph node metastases and developed nmCRPC after only 9-month combined use of ADT and antiandrogen, so limited efficacy of ARAT could be expected. Given the disappointing response rate, the financial burden and the lack of availability of apalutamide and enzalutamide in China, we had to seek a new treatment strategy.

We tested the expression status of several tumor markers by IHC staining using his pre-treatment prostate biopsy samples, as he refused second biopsy in 9 months. We were not surprised to find the biopsy specimens were positive for $\mathrm{AR}$ and negative for ARV7, since his tumor was hormone sensitive at the beginning of ADT, while ARV7 is seldom expressed in hormone-sensitive prostate cancer (HSPC) (11). It was also worth noting that MUC1 was partially positive and BRCA1 was negative in this IHC staining. Previous study suggests that MUC1 is aberrantly expressed in prostate cancer with aggressive pathological features and poorer clinical outcomes, including larger tumor volume as well as nodal involvement, and early disease progression (12). These findings could, in part, explain the bulky lymph node metastases and early ADT resistance in our case. BRCA1 is a regulator of prostate cancer radiosensitivity, which has been reported that knockdown of BRCA1 can lead to significant radiosensitization of prostate cancer cells in vitro (13). Thus, prostate cancer patients with BRCA1 negative expression may have a good response to radiotherapy.

Individuals with rapid progression to CRPC may have poor response to hormone therapies but not to chemotherapy. Docetaxel has been widely used in mCRPC over a decade and is recommended by NCCN guideline. For high risk localized patients, including those with 


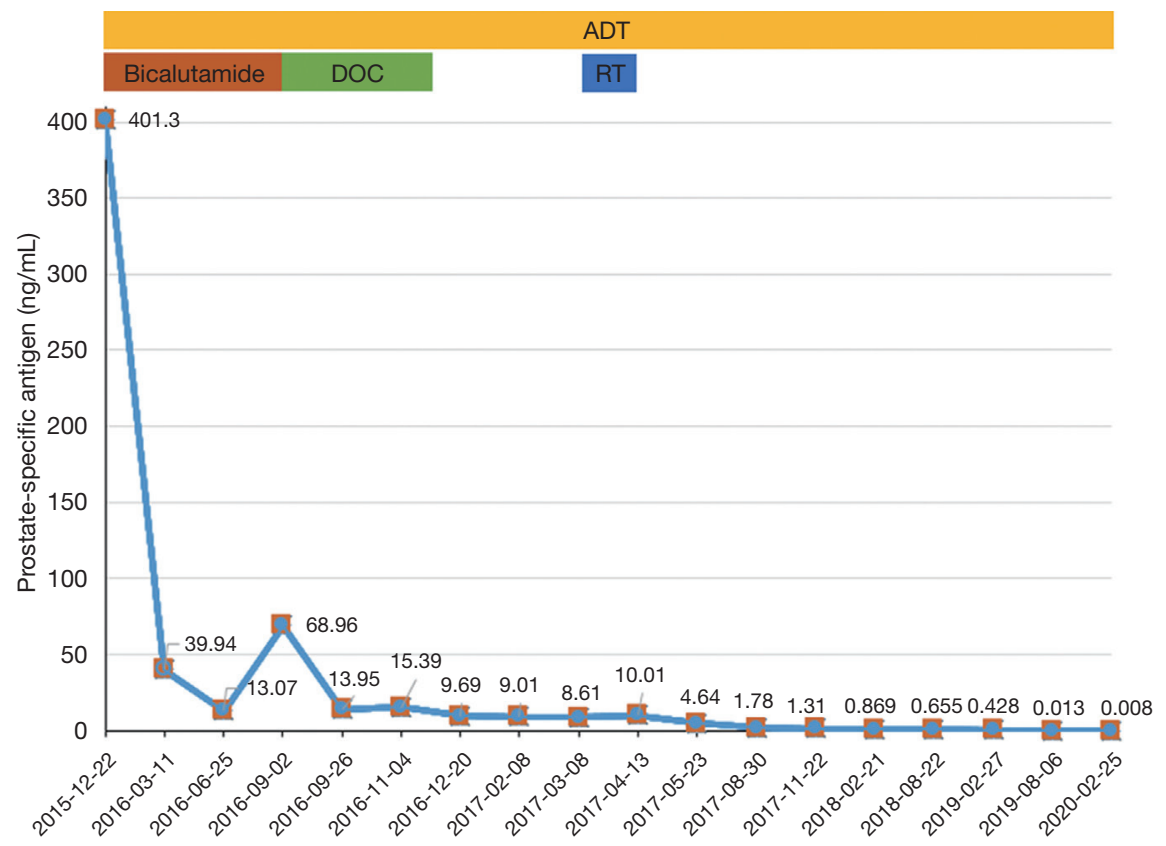

Figure 5 Prostate-specific antigen (PSA) changes from the initial treatment. ADT, androgen deprivation therapy; DOC, docetaxel; RT, radiotherapy.

Sustaining Goserelin 10.8 mg every 84 days

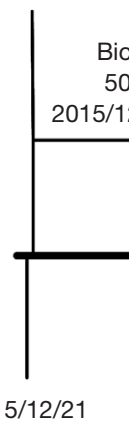

Intermittent dysuria

for 3 years.

Diagnosed with

prostate cancer
Bicalutamide

$50 \mathrm{mg}$ daily

$15 / 12 / 22-2016 / 9 / 2$

Docetaxel $75 \mathrm{mg} / \mathrm{m}^{2}$ every 21 days for 6 cycles 2016/9/3-2016/12/17
IGRT-VMAT to the prostate, the enlarged lymph nodes and the whole pelvis 2017/3/8-2017/4/13
Regular check by serum PSA every 3 months for 1 year then every 6 months and pelvic MRI every 1year till now

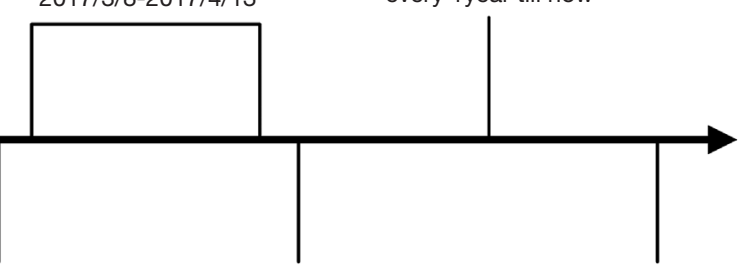

$2020 / 2 / 25$

2017/2/8

PSA decreased. Metastatic lymph nodes continuously shrank
2017/5/23

PSA decreased gradually
PSA decreased to $0.008 \mathrm{ng} / \mathrm{mL}$. Latest MRI showed no tumor in the prostate and the previous bulky lymph node shrunk to only $8 \mathrm{~mm}$ without sign of metastases

A 50-year history of smoking and alcohol drinking (250 g daily); family hitory unremarkable

Figure 6 The timeline picture of this case. nmCRPC, non-metastatic castration-resistant prostate cancer; NGS, next-generation sequence; PSA, prostate-specific antigen; IGRT-VMAT, image-guided volumetric modulated arc radiotherapy; MRI, magnetic resonance imaging. 
Table 1 Studies (more than 50 cases) on radiotherapy for nmCRPC

\begin{tabular}{lccccl}
\hline Author, year & $\begin{array}{c}\text { Total } \\
\text { no. }\end{array}$ & $\begin{array}{c}\text { No. of } \\
\text { No/N1 }\end{array}$ & $\begin{array}{c}\text { RT dose } \\
\text { (Gy) }\end{array}$ & $\begin{array}{c}\text { Median } \\
\text { follow-up (m) }\end{array}$ & Outcomes \\
\hline White et al. (16), 2015 & 51 & $51 / 0$ & 50 & 54 & 3y FFLF: 85\%; 5y FFLF: 81\%; 5y CSS: 65\%; 5y OS: 35\% \\
Akimoto et al. (17), 2003 & 53 & $53 / 0$ & 69 & 35 & 3y CSS: 94\%; 3y CFFS: 78\%; 5y CSS: 87\%; 5y CFFS: 56\% \\
Sasaki et al. (18), 2009 & 140 & $84 / 28$ & 66 & 20.7 & 5y OS: 48.1\%; 5y CPFS: 36.7\% \\
Ogawa et al. (19), 2008 & 84 & $58 / 10$ & 66 & 26.9 & 3y OS: 67\%; 3y LCR: 93\%; 3y PFS: 61\% \\
Nakamura et al. (20), 2004 & 61 & $45 / 15$ & 60 & 17 & 5y OS: $51.6 \%$; 5y PFS: 43.5\%
\end{tabular}

m, month; y, year; FFLF, free from local failure; CSS, cancer-specific survival; OS, overall survival; CFFS, clinical failure-free survival; RT, radiotherapy; LCR, locoregional control rate; CPFS, clinical progression-free survival; PFS, progression-free survival.

regional lymph nodes metastasis, the use of docetaxelbased chemotherapy before definitive local therapy can improve relapse-free survival (4). However, for nmCRPC, there is only one retrospective study shows that the early use of docetaxel can prolong median OS (6). Toxicity is one of the major concerns regarding docetaxel, but some previous studies reveal that docetaxel is safe and its toxicity will disappear in 1 year without lifelong influence (14). Despite our patient is of advanced age, his chemotherapyrelated polymorphism analysis suggests low docetaxelinduced toxicity. Indeed, the patient tolerated well during chemotherapy and after 6 cycles of docetaxel, his total PSA dropped sharply from pre-chemotherapy value of 68.96 to $8.61 \mathrm{ng} / \mathrm{mL}$. Thus, using docetaxel in the treatment of nmCRPC prior to ARAT in carefully selected patients might provide a new train of thought.

The addition of radiotherapy to ADT could significantly prolong survival in patients with lymph node metastases (3), making it indispensable in node-positive disease in current practice. Local radiotherapy for nmCRPC may hopefully provide excellent local-regional control, delay biochemical recurrence (15). Despite the high-level prospective evidence is still lacking, several retrospective studies have supported the application of radiotherapy in nmCRPC (Table 1).

As in our case, while nmCRPC with bulky regional lymph node metastases is especially rare and refractory, satisfactory tumor control has been achieved by the combined use of ADT, docetaxel and radiotherapy. However, the evidence is limited with only one case currently, and further prospective study is urgently needed to confirm the role and timing of radiotherapy in nmCRPC.

\section{Conclusions}

Prostate cancer patients with regional lymph node metastases is highly heterogeneous, and those with bulky tumor are highly malignant and potentially rapidly develop to CRPC. Molecular analysis may be helpful to better understand internal biological characteristics. Individualized and multimodal therapy, including intensification of systemic therapy and timely application of radiotherapy could be suggested in such cases.

\section{Patient perspective}

"I was once very anxious about my disease and worried about the possible toxicities of hormone therapy, chemotherapy and radiotherapy. But after receiving these treatments, I feel no obvious discomfort. Now, there has been more than 4 years since I was diagnosed with advanced prostate cancer, and I feel great and very satisfied with my condition."

\section{Acknowledgments}

Funding: None.

\section{Footnote}

Reporting Checklist: The authors have completed the CARE reporting checklist. Available at http://dx.doi.org/10.21037/ tau-20-725

Conflicts of Interest: All authors have completed the ICMJE uniform disclosure form (available at http://dx.doi. org/10.21037/tau-20-725). The authors have no conflicts of interest to declare.

Ethical Statement: The authors are accountable for all aspects of the work in ensuring that questions related to the accuracy or integrity of any part of the work are 
appropriately investigated and resolved. Written informed consent was obtained from the patient for publication of this case report and any accompanying images.

Open Access Statement: This is an Open Access article distributed in accordance with the Creative Commons Attribution-NonCommercial-NoDerivs 4.0 International License (CC BY-NC-ND 4.0), which permits the noncommercial replication and distribution of the article with the strict proviso that no changes or edits are made and the original work is properly cited (including links to both the formal publication through the relevant DOI and the license). See: https://creativecommons.org/licenses/by-nc-nd/4.0/.

\section{References}

1. Bray F, Ferlay J, Soerjomataram I, et al. Global cancer statistics 2018: GLOBOCAN estimates of incidence and mortality worldwide for 36 cancers in 185 countries. CA Cancer J Clin 2018;68:394-424.

2. Zheng RS, Sun KX, Zhang SW, et al. Report of cancer epidemiology in China, 2015. Zhonghua Zhong Liu Za Zhi 2019;41:19-28.

3. James ND, Spears MR, Clarke NW, et al. FailureFree Survival and Radiotherapy in Patients With Newly Diagnosed Nonmetastatic Prostate Cancer: Data From Patients in the Control Arm of the STAMPEDE Trial. JAMA Oncol 2016;2:348-57.

4. Rosenthal SA, Hu C, Sartor O, et al. Effect of Chemotherapy With Docetaxel With Androgen Suppression and Radiotherapy for Localized HighRisk Prostate Cancer: The Randomized Phase III NRG Oncology RTOG 0521 Trial. J Clin Oncol 2019;37:1159-68.

5. Attar RM, Takimoto CH, Gottardis MM. Castrationresistant prostate cancer: locking up the molecular escape routes. Clin Cancer Res 2009;15:3251-5.

6. Ito K, Kimura T, Onuma H, et al. Does docetaxel prolong survival of patients with non-metastatic castration-resistant prostate cancer? Prostate 2018;78:498-505.

7. Riley DS, Barber MS, Kienle GS, et al. CARE guidelines for case reports: explanation and elaboration document. J Clin Epidemiol 2017;89:218-35.

8. Halabi S, Kelly WK, Ma H, et al. Meta-Analysis Evaluating the Impact of Site of Metastasis on Overall Survival in Men With Castration-Resistant Prostate
Cancer. J Clin Oncol 2016;34:1652-9.

9. Mori K, Kimura T, Ito K, et al. Earlier use of androgen receptor-axis-targeted drugs may improve overall survival in patients with non-metastatic castration-resistant prostate cancer. Prostate 2018;78:766-72.

10. Loriot Y, Eymard JC, Patrikidou A, et al. Prior long response to androgen deprivation predicts response to next-generation androgen receptor axis targeted drugs in castration resistant prostate cancer. Eur J Cancer 2015;51:1946-52.

11. Welti J, Rodrigues DN, Sharp A, et al. Analytical Validation and Clinical Qualification of a New Immunohistochemical Assay for Androgen Receptor Splice Variant-7 Protein Expression in Metastatic Castration-resistant Prostate Cancer. Eur Urol 2016;70:599-608.

12. Genitsch V, Zlobec I, Thalmann GN, et al. MUC1 is upregulated in advanced prostate cancer and is an independent prognostic factor. Prostate Cancer Prostatic Dis 2016;19:242-7.

13. Gorodetska I, Lukiyanchuk V, Peitzsch C, et al. BRCA1 and EZH2 cooperate in regulation of prostate cancer stem cell phenotype. Int J Cancer 2019;145:2974-85.

14. Fizazi K, Lesaunier F, Delva R, et al. A phase III trial of docetaxel-estramustine in high-risk localised prostate cancer: a planned analysis of response, toxicity and quality of life in the GETUG 12 trial. Eur J Cancer 2012;48:209-17.

15. Henkenberens C, Merseburger AS, Bengel F, et al. Radiotherapy for isolated lymph node metastases in patients with locally advanced prostate cancer after primary therapy. World J Urol 2016;34:1239-45.

16. White R, Khor R, Bressel M, et al. Efficacy of highdose palliative radiotherapy for localised, castrationresistant prostate cancer. Clin Oncol (R Coll Radiol) 2015;27:16-21.

17. Akimoto T, Kitamoto Y, Saito J, et al. External beam radiotherapy for clinically node-negative, localized hormone-refractory prostate cancer: impact of pretreatment PSA value on radiotherapeutic outcomes. Int J Radiat Oncol Biol Phys 2004;59:372-9.

18. Sasaki T, Nakamura K, Ogawa K, et al. Radiotherapy for patients with localized hormone-refractory prostate cancer: results of the Patterns of Care Study in Japan. BJU Int 2009;104:1462-6.

19. Ogawa K, Nakamura K, Sasaki T, et al. External beam 
radiotherapy for clinically localized hormone-refractory prostate cancer: clinical significance of Nadir prostatespecific antigen value within 12 months. Int J Radiat Oncol Biol Phys 2009;74:759-65.

Cite this article as: Mai L, Li Y, Yang P, Zhang Z, Wu J, Zhou F, Liu Y, He L. Successful management of prostate cancer with bulky pelvic lymph node metastases after rapid development of castration-resistant prostate cancer: a case report with review of the literature. Transl Androl Urol 2020;9(3):1428-1436. doi:10.21037/tau-20-725
20. Nakamura K, Teshima T, Takahashi Y, et al. Radiotherapy for localized hormone-refractory prostate cancer in Japan. Anticancer Res 2004;24:3141-5. 